\title{
Calcium Silicate: The Multi-Faceted Material
}

\author{
Dr. Seema D. Pathak ${ }^{1}$, Dr. Pradnya V. Bansode ${ }^{2}$, Dr. Anjali Pandey ${ }^{3}$ \\ ${ }^{1}$ Associate Professor, Department of Conservative Dentistry and Endodontics, GDC \& Hospital, Aurangabad, MUHS, India
}

${ }^{2}$ Professor and Head of Department, Department of Conservative Dentistry and Endodontics, GDC \& Hospital, Aurangabad, MUHS, India

${ }^{3}$ Final Year Post-Graduate Student, Department of Conservative Dentistry and Endodontics, GDC \& Hospital, Aurangabad, MUHS, India

\begin{abstract}
Calcium Silicate is a material used widely Endodontics starting from used as a pulp capping agent to root-end filling material. This can be credited to the invention of MTA, since then many formulations have been tried and are still under research to enhance the success rate of these procedures.
\end{abstract}

Keywords: Calcium silicate, Endodontics, MTA, root-end filling

\section{Introduction}

Calcium silicate is one of the most commonly used materials in dentistry. Because of its minimal reactivity and biocompatibility especially with hard tissue, it is gaining popularity amongst practitioners and researchers. One of the most important inventions related with it was Mineral Trioxide Aggregate (MTA). After this revolutionary invention many others materials were invented. It is widely used in all the aspects of dentistry, while this review article mainly focuses on the various formulations used in Endodontics.

\section{Calcium Silicate in Endodontics}

\subsection{Mineral Trioxide Aggregate}

Mineral Trioxide Aggregate (MTA) was introduced by Mohmoud Torabinejad at Loma Linda University, California, USA in 1993 and was given approval for endodontic use by the U.S. Food and Drug Administration in 1998. MTA is available in two types based on the color known as gray and white MTA. Scanning electron microscopy (SEM) and electron probe microanalysis characterized the differences between GMTA and WMTA and found that the major difference between GMTA and WMTA is in the concentrations of $\mathrm{Al}_{2} \mathrm{O}_{3}, \mathrm{MgO}$ and $\mathrm{FeO}$. [1,2]

Table 1: Chemical compositions of GMTA and WMTA [Adapted from Asgary et al. (2005)] [1]

\begin{tabular}{|c|c|c|}
\hline Chemical compound & $\begin{array}{c}\text { GMTA } \\
\text { (wt\%) }\end{array}$ & $\begin{array}{c}\text { WMTA } \\
\text { (wt\%) }\end{array}$ \\
\hline Calcium oxide $(\mathrm{CaO})$ & 40.45 & 44.23 \\
\hline Silicon dioxide $(\mathrm{SiO} 2)$ & 17.00 & 21.20 \\
\hline Bismuth trioxide $(\mathrm{Bi} 2 \mathrm{O} 3)$ & 15.90 & 16.13 \\
\hline Aluminium oxide $(\mathrm{Al2O} 3)$ & 4.26 & 1.92 \\
\hline Magnesium oxide $(\mathrm{MgO})$ & 3.10 & 1.35 \\
\hline Sulfur trioxide $(\mathrm{SO} 3)$ & 0.51 & 0.53 \\
\hline Chlorine $(\mathrm{Cl})$ & 0.43 & 0.43 \\
\hline Ferrous oxide $(\mathrm{FeO})$ & 4.39 & 0.40 \\
\hline Phosphorus pentoxide $(\mathrm{P} 2 \mathrm{O} 5)$ & 0.18 & 0.21 \\
\hline Titanium dioxide $(\mathrm{TiO} 2)$ & 0.06 & 0.11 \\
\hline Carbonic acid $(\mathrm{H} 2 \mathrm{O}+\mathrm{CO} 2)$ & 13.72 & 14.49 \\
\hline
\end{tabular}

The powder water ratio for MTA should be 3:1(P: W). Mixing can be done on paper pad or on a glass slab using a plastic or metal spatula to achieve putty like paste consistency. This mix should be covered with moistened cotton pellet to prevent dehydration of mix. [3]

Immediately after mixing MTA has a $\mathrm{pH}$ of 10.2. After 3 hours of setting the $\mathrm{pH}$ increased to 12.5 . The $\mathrm{pH}$ of set MTA is almost similar to calcium hydroxide. The mixing time should be less than 4 minute and setting time is about 2 hours and 55 minutes and 2 hours and 20 minutes for grey MTA and white MTA, respectively. MTA being hydrophilic requires moisture to set. Presence of moisture during setting improves the flexural strength of the set cement. Therefore, it is advised to place a wet cotton pellet over the MTA in the first visit followed by replacement by a permanent restoration at the second visit. The long setting time is one of the drawbacks of MTA because of it should not be applied in 1 visit. Inter-appointment moist cotton palate is required till the final setting of MTA.

MTA powder must be kept tightly closed to avoid degradation by moisture. The mixing time is critical as, if the mixing time is prolonged; it results in dehydration of the mix.. MTA may be placed into the desired location using ultrasonic condensation, plugger, paper point or specially designed carriers and messing gun. [3]

2.1.1 Chemical, Physical, and Mechanical Properties of MTA [1-5] : Compressive strength

The compressive strength at 24 hours $40.0 \mathrm{MPa}$ and at 21 days 67.3MPa; and in comparison between GMTA and WMTA result showed that compressive strength of Gray MTA > White MTA.

\subsubsection{Radio-opacity}

MTA has comparable radiodensity as Zinc Oxide Eugenol and it is less radio opaque than Super EBA, IRM, guttapercha or amalgam.

\subsubsection{Solubility}

The set MTA shows no signs of solubility. But, if more water is used during mixing the MTA it may results into increased solubility. Also set MTA when exposed to water it releases 


\section{International Journal of Science and Research (IJSR) \\ ISSN (Online): 2319-7064}

Index Copernicus Value (2015): 78.96 | Impact Factor (2015): 6.391

calcium hydroxide $(\mathrm{CaOH} 2)$. $\mathrm{CaOH} 2$ might be responsible for its cementogenesis-inducing property. During setting reaction if mix is exposed to acidic environment it does not interfere in the setting.

\subsubsection{Marginal adaptation and sealing ability}

MTA has excellent sealing ability which may occur because MTA expands during setting reaction. In presence of moist environment sealing ability of MTA is enhanced due to the setting expansion so it is been suggested that a moistened cotton pellet should be placed in contact with MTA before placement of the permanent restoration. About 4-mm thickness of MTA is sufficient to ensure a good sealing.

\subsubsection{Antibacterial and antifungal property}

According to some authors, MTA has antibacterial effect especially against Enterococcus faecalis and Streptococcus sanguis.

\subsubsection{Reaction with other dental materials}

MTA does not react or interfere with any other restorative material. When GIC or composite resins placed over MTA it doesn't affects the setting reaction. But residual calcium hydroxide may interfere with the adaptation of MTA to dentinal wall. This results in reduced sealing ability which occurs either by a mechanical obstacle of $\mathrm{CaOH}_{2}$ particle by chemically reaction with MTA.

\subsubsection{Biocompatibility}

MTA is not mutagenic and less cytotoxic. Also MTA is well tolerated by the tissues and biocompatible. It has good interaction with periapical and periradicular tissues. MTA has potential effect on cell viabilities collagen release mechanism. The property of MTA to produce interleukin and also offers a biologically active substrate for bone cells.

\subsubsection{Tissue regeneration}

MTA has potential to activate the cementoblasts and eventually cementum production. MTA also allows the overgrowth of PDL fiber over its surface and helps in elimination of clinical symptoms bone healing. These properties of MTA determine it as a potential regenerative material.

\subsubsection{Mineralization}

MTA forms dentin bridge similar to calcium hydroxide $\left(\mathrm{CaOH}_{2}\right)$. The tricalcium oxide content of MTA interacts with tissue fluids and form $\mathrm{CaOH}_{2}$, resulting in hard-tissue creation in a similar manner to that of $\mathrm{CaOH}_{2}$. The bridge formation is relatively faster, with good structural integrity than with $\mathrm{CaOH}_{2}$ and it stimulates reparative dentin formation along with maintaining the integrity of the pulp.

\subsubsection{Clinical Applications of MTA}

1. In Primary teeth:

i. Pulp capping

ii. Pulpotomy

iii. Root canal filling

iv. Furcation perforation repair

v. Resorption repair
2. In Permanent teeth:

i. Pulp capping

ii. Partial pulpotomy

iii. Perforation repair - Apical, lateral, furcation

iv. Resorption repair - External and internal

v. Repair of fracture - Horizontal and Vertical

vi. Root end filling

vii. Apical barrier for tooth with necrotic pulps and open apex

viii. Coronal barrier for regenerative endodontics

ix. Root canal sealer

\subsection{Endosequence [6]}

Endosequence root repair material (ERRM) has been developed as ready-to-use. This premixed bioceramic materials recommended for perforation repair, apical surgery, apical plug, and pulp capping. The manufacturer stated that the moisture present in the dentinal tubules is adequate to allow the material to set. ERRM is stated by the manufacturer to bond to adjacent dentine, to have no shrinkage, and to be highly biocompatible, hydrophilic, radiopaque, and antibacterial due to a high $\mathrm{pH}$ during setting. The major advantages of this material are improved handling characteristics over traditional MTA and the delivery of a consistent product with each application.

\subsubsection{Chemical Composition and Characteristics}

ERRM is composed of calcium silicates, monobasic calcium phosphate, zirconium oxide, tantalum oxide, proprietary fillers and thickening agents. The material has nanosphere particles with a maximum diameter of $1 \times 10^{-3} \mu \mathrm{m}$ that allow for the material to enter dentinal tubules, be moistened by dentine liquid, and create a mechanical bond upon setting. This material has been manufactured to overcome some of the difficult handling characteristics of MTA.

\subsubsection{Bioactivity}

This material is bioactive due to its ability to form a hydroxyapatite or apatite-like layer on its surface when it comes in contact with phosphate-containing fluids.

\subsubsection{Biocompatibility and Cytotoxicity}

ERRM is able to bond to adjacent dentine, to have no shrinkage, and to be highly biocompatible. AlAnezi et al. used cultured mouse fibroblast cells to determine the cytotoxicity of ERRM as compared with gray and white MTA and found that both set and fresh samples showed no significant cell viability differences.

\subsubsection{Sealing Ability}

Hirschberg et al. compared the sealing ability of MTA to the sealing ability of ERRM using a bacterial leakage model. They concluded that Samples in the ERRM group leaked significantly more than samples in the MTA group.

\subsubsection{Antibacterial Activity}

Lovato and Sedgley investigated the antibacterial activity of ERRM against Enterococcus faecalis. They found that ERRM and white ProRoot MTA demonstrated similar antibacterial efficacy against clinical strains of E. faecalis.

Volume 6 Issue 12, December 2017 


\section{International Journal of Science and Research (IJSR) \\ ISSN (Online): 2319-7064}

Index Copernicus Value (2015): 78.96 Impact Factor (2015): 6.391

This research again validated earlier studies that found ERRM displayed similar in vitro biocompatiblity to MTA.

Additionally, other study found that the ERRM had cell viability similar to Gray and White MTA in both set and fresh conditions.

\subsection{Biodentine [6]}

Calcium silicate-based material has been recently developed to overcome some of shortcomings of MTA, which are difficult handling, long setting time, and potential discoloration. Calcium silicate-based material, which called Biodentine, was declared by dental materials manufacturer Septodont in September of 2010, and made available in January of 2011. This material is new biologically active cement which has dentine-like mechanical properties. It also can be used as a dentine replacement in the tooth crown and root region. Compared to MTA, Biodentine handles easily and needs much less time for setting. Unlike other Portland cement-based products, it is sufficiently stable so that it can be used both for pulp protection and temporary fillings. This is why the manufacturer recommends to fill the entire cavity completely with Biodentine in a first step and to reduce it to a base/dentine substitute level in a second visit one week to 6 months later before definitive restoration. For successful capping it is, however, important to seal the cavity against bacterial invasion in a one-stage procedure.

\subsubsection{Clinical Applications}

Biodentine has many applications in Dentistry such as crown and root dentine repair treatment, repair of perforations or resorptions, apexification and root-end fillings. The material can also be used in class II fillings as a temporary enamel substitute and as permanent dentine substitute in large carious lesions. The manufacturer claimed about the biocompatibility and the bioactivity of the material, which is important when used as indirect and direct pulp capping and pulpotomy. Furthermore, it preserves pulp vitality and promotes its healing process. Both Biodentine and MTA may modify the proliferation of pulp cell lines. Their effects may fluctuate over time, depending on the cell line considered. The observed similarity between Biodentine and MTA validates the indication for direct pulp capping claimed by the manufacturers.

\subsubsection{Chemical Composition and Characteristics}

Biodentine consists of a powder in a capsule and liquid in a pipette. The powder mainly contains tricalcium and dicalcium silicate, the principal component of Portland cement, as well as calcium carbonate. Zirconium dioxide serves as contrast medium. The liquid consists of calcium chloride in aqueous solution with an admixture of polycarboxylate. Once mixed, Biodentine sets in approximately 12 minutes. The consistency of Biodentine is similar to that of phosphate cement.

Dental materials based on tricalcium silicate cement and MTA release minimal quantities of trace elements when in contact with simulated body fluids. Biodentine stimulates dentine regeneration by inducing odontoblast differentiation from pulp progenitor cells. Histologically, the bioactive tricalcium silicate demonstrated the ability to induce odontoblast differentiation from pulp progenitor cells. The resulting mineralized matrix had the molecular characteristics of dentine..

\subsubsection{Sealing Ability and Success}

Biodentine is stronger mechanically, less soluble and produces tighter seals. This qualifies it for avoiding three major drawbacks of calcium hydroxide, i.e. material resorption, mechanical instability and the resultant failure of preventing microleakages. Biodentine causes alkaline corrosion on the hard tissue, which leads to a so-called "mineral interaction zone". Due to remodelling processes, the sealing of the dentine by Biodentine improves in the course of time.

\subsubsection{Antibacterial Properties}

During the setting phase of Biodentine, calcium hydroxide ions are released from the cement. This results in a $\mathrm{pH}$ of about 12.5 and a basification of the surroundings. This high $\mathrm{pH}$ inhibits the growth of microorganisms and can disinfect the dentine.

\subsubsection{Morphological and Chemical Characteristics of the Interface between Human Dentine and Biodentine}

The morphological and chemical characteristics of the interface between human dentine and new calcium silicate based dental cement were investigated. The dentine Biodentine interface is dynamic and interactive; that is manifested by water movement between the two substrates, and hydrated cement diffusion into the dentine, accompanied by microstructural changes.

\subsection{Bioaggregate [3, 6]}

BioAggregate is new generation of a root canal repair filling material. The manufacturer claimed that BioAggregate is produced under controlled conditions to form contamination free ceramic nano-particles. According to manufacturer, BioAggregate is developed as a result of utilizing the advanced science of nano-technology to produce ceramic particles that, upon reaction with water produce biocompatible and aluminum-free ceramic biomaterials.

BioAggregate has excellent handling characteristics after mixing with water, which aids in a repair process of the affected tooth. BioAggregate's radiopacity properties, convenient setting and hardening time and easy workability and handling properties make it an ideal root canal filling material. As stated by manufacturer, the working time of BioAggregate is at least 5 minutes. Upon mixing a thick paste-like mixture is formed. If additional working time is required, simply cover the mixture with a moist gauze sponge while unattended.

\subsubsection{Chemical Composition and Characteristics}

As stated by manufacturer, the composition of BioAggregate is tricalcium silicate, dicalcium silicate, tantalum pentoxide, and calcium phosphate monobasic. To provide radiopacity, tantalum pentoxide is used in BioAggregate rather than the bismuth oxide used in MTA. This was confirmed by Park et al., when examined the chemical differences between white MTA and BioAggregate in both powder and set forms using X-ray diffraction. The results showed that MTA and BioAggregate have a similar chemical composition with

\section{Volume 6 Issue 12, December 2017}




\section{International Journal of Science and Research (IJSR) \\ ISSN (Online): 2319-7064}

Index Copernicus Value (2015): 78.96 | Impact Factor (2015): 6.391

some differences. BioAggregate contains a significant amount of tantalum oxide instead of bismuth oxide. In both groups, similar peaks were observed in the set and powder form, but sharper and stronger peaks were observed in the powder samples. Furthermore, Camilleri et al. determined the elemental constitution and investigated the total and leachable arsenic, chromium and lead in Portland cement, pure tricalcium silicate, Biodentine, BioAggregate and MTA. They concluded that dental materials based on tricalcium silicate cement and MTA release minimal quantities of trace elements when in contact with simulated body fluids. The results of acid extraction could be affected by nonspecific matrix effects by the cement.

The BioAggregate powder promotes a complicated set of reactions upon mixing with BioA Liquid (deionized water), which leads to the formation of a nano-composite network of gellike calcium silicate hydrate intimately mixed with hydroxyapatite bioceramic, and forms a hermetic seal when applied inside the root canal. This is also supported by Grech et al., who investigated the composition of materials and leachate of a hydrated prototype cement composed of tricalcium silicate and radiopacifier. They then compared this to other tricalcium silicate-based cements which are Biodentine and BioAggregate to assess whether the additives in the proprietary brand cements affect the hydration of the materials. They used IRM as a standard root-end filling material. They found that Biodentine and BioAggregate resulted in the formation of calcium silicate hydrate and calcium hydroxide, which was leached in solution. The hydrated materials were composed of a cementitous phase that was rich in calcium and silicon and a radiopacifying material. Biodentine included calcium carbonate; Whereas BioAggregate included silica and calcium phosphate in the powders. IRM was composed of zinc oxide interspersed in a matrix of organic material.

\subsubsection{Clinical Applications}

The manufacturer claimed that BioAggregate is a biocompatible pure white powder composed of ceramic particles. Upon mixing, the hydrophilic BioAggregate Powder promotes cementogenesis and forms a hermetic seal inside the root canal. It is effective in clinically blocking the bacterial infection, its ease of manipulation and superior quality makes BioAggregate the most innovative and unique root canal repair material. According to manufacturer, the BioAggregate is indicated for repair of root perforation, repair of root resorption, root end filling, apexification, and pulp capping. Shokouhinejad et al. evaluated the bioactivity of BioAggregate, ERRM, and MTA. They concluded that exposure of MTA, BioAggregate and ERRM to PBS resulted in precipitation of apatite crystalline structures that increased over time. This suggested that the tested materials are bioactive.

\subsubsection{Sealing Ability and Success}

El Sayed and Saeed evaluated and compared sealing ability of BioAggregate versus amalgam, IRM and MTA. They reported that BioAggregate has high sealing ability. They authors considered that utilizing the BioAggregate as alternative to MTA.

\subsubsection{Properties of Bioaggregate}

Tuna et al. assessed the long-term fracture resistance of human immature permanent teeth filled with BioAggregate, MTA and calcium hydroxide. They suggested that BioAggregate-filled immature teeth demonstrate higher fracture resistance than other groups at 1year. Considering the long-term risk of cervical root fracture associated with immature teeth, the use of BioAggregate as a root canal filling material appears to be the most advantageous of the materials tested. Grech et al. investigated the physical properties of prototype radiopacified tricalcium silicate cement, Bioaggregate and Biodentine. IRM was used as a control. They reported that the addition of admixtures to tricalcium silicate-based cements affects the physical properties of the materials. Research suggests that the high $\mathrm{pH}$ and released calcium ions are required for a material to stimulate mineralization in the process of hard tissue healing.

\subsection{Light-Cure MTA [5]}

An experimental light-cure MTA has been developed to have similar properties to MTA and also better working properties. Although this experimental material apparently presents positive characteristics, there are very few studies regarding its biocompatibility.

\subsection{CER [4]}

Recently, a new MTA formulation (Cimento Endodontico Rapido or Fast Endodontic Cement) composed of Portland cement in a gel with water, barium sulphate, and an emulsifier, whose function is to improve handling properties, has been also tried. One research evaluated the rat subcutaneous tissue response to Fast Endodontic cement (CER, Cimento Endodontico Rapido) and Angelus MTA. Results showed that both materials were biocompatible and stimulated mineralization.

\section{7 iROOT BP Plus Bioceramic Putty [7]}

iRoot BP Plus (Innovative BioCeramix Inc., Vancouver, Canada) is fully laboratory-synthesized, water-based bioceramic cement. It claims to be a more convenient reparative material, because it is a ready to-use white hydraulic premixed formula.70A current study to verify in vitro cytocompatibility of iRoot BP Plus bioceramic putty concluded that iRoot and MTA were biocompatible and did not induce critical cytotoxic effects.

\subsection{Novel Root-End Filling Material [6]}

A novel resin based root-end filling material (termed New resin cement, NRC) has been introduced. NRC is a powder and liquid system. The liquid is composed of hydroxyethylmethacrylate, benzoyl peroxide, toluidine, and toluenesulfinate. And the powder is made of calcium oxide, calcium silicate, and triphenylbismuth carbonate. One study determined the cytotoxicity of NRC and concluded that the initial biocompatibility results of NRC are favorable for a root-end filling material. A recent in vivo study concluded that NRC shows moderately higher inflammatory reaction 


\section{International Journal of Science and Research (IJSR) \\ ISSN (Online): 2319-7064}

Index Copernicus Value (2015): 78.96 | Impact Factor (2015): 6.391

than MTA however, the calcium reservoir capability of NRC may contribute to mineralization of the tissues.

\subsection{Experimental Calcium Aluminosilicate Based Materials [6]}

\subsubsection{EndoBinder}

A new calcium aluminate-based endodontic cement, called EndoBinder (Binderware, Sao Carlos, SP, Brazil), has been developed with the intention of preserving the properties and clinical applications of MTA eliminating its negative characteristics. EndoBinder is produced with high levels of purity, eliminating traces of free magnesium oxide $(\mathrm{MgO})$ and calcium oxide $(\mathrm{CaO})$, which are responsible for the undesired expansion of the material, and ferric oxide (Fe2O3), which is responsible for tooth darkening. Among recent materials, EndoBinder presented satisfactory tissue reaction, it was biocompatible when tested in subcutaneous tissue of rats.

\subsubsection{Generex A}

Generex A (Dentsply Tulsa Dental Specialties, Tulsa, OK, USA) is a calcium-silicate-based material that has some similarities to ProRoot MTA but is mixed with unique gels instead of water used for MTA. Generex A material has very different handling properties in comparison to MTA. Generex A mixes to a dough-like consistency, making it easy to roll into a rope-like mass similar to intermediate restorative material.

\subsubsection{Capasio}

Capasio (Primus Consulting, Bradenton, FL, USA) is composed primarily of bismuth oxide, dental glass, and calcium alumino-silicate with a silica and polyvinyl acetatebased gel. A recent study found that Capasio and MTA promote apatite deposition when exposed to synthetic tissue fluid thus had the mineralization capacity. The same researchers also concluded that when used as a root-end filling material, Capasio is more likely to penetrate dentinal tubules. Another study compared Generex A, Generex B, Capasio along with Ceramicrete-D (magnesium phosphate based) using primary osteoblasts. Generex A was the only new generation endodontic material that supported primary osteoblast growth. No material besides MTA facilitated nodule formation. Only Generex A and MTA allowed cell growth and proliferation throughout the experiment.

\subsubsection{Quick-Set}

Recently, Capasio powder has been refined and renamed as Quick-Set (Primus Consulting), and the cationic surfactant was removed from the liquid gel component, which was thought to interfere with cytocompatibility. In a contemporary research using odontoblast-like cells, Quick-Set and MTA exhibited similar cytotoxicity profiles. They possess negligible in vitro toxicologic risks after timedependent elution of toxic components.

\subsubsection{Novel root-end filling material using epoxy resin and Portland cement (EPC)}

EPC, a novel composite made from a mixture of epoxy resin and Portland cement, was found to be a useful material for root-end filling, with favorable radio-opacity, short setting time, low microleakage, and clinically acceptable low cytotoxicity.

\subsubsection{Iron-free partially stabilized cement}

Partial stabilized cement (PSC) is an innovative material prepared to address some of the drawbacks of MTA. Portland cement-based PSC with $\mathrm{Zn}$ was synthesized by replacing iron nitrate using one-step sol-gel process. The physical properties and biocompatibility of PSCZn were found to be favourable as an ideal root-end filling material.

\subsection{CEM [8]}

A novel endodontic cement named calcium-enriched mixture (CEM) cement was introduced to dentistry in 2006 as an endodontic filling material. The physical properties of this biomaterial, such as flow, film thickness, and primary setting time are favorable. It has the ability to promote hydroxyapatite formation in saline solution and might promote the process of differentiation in stem cells and induce hard tissue formation. It also possesses ability to set in aqueous environments with shorter setting time than MTA and sealing ability comparable to MTA. The clinical uses of the CEM cement are similar to MTA. CEM cement has demonstrated similar results to MTA when used as pulp capping agent or furcation perforation repair. It has also shown favorable results in pulpotomy of permanent molar teeth with established irreversible pulpitis, and in management of internal root resorption. Furthermore, this material has an antibacterial effect comparable to calcium hydroxide and better than MTA or Portland cement (PC).

\subsubsection{Composition and mechanism of action}

CEM cement is composed of different calcium compounds. The major components of the powder are $\mathrm{CaO}(51.75 \%)$, $\mathrm{SO}_{3}$ (9.53\%), $\mathrm{P}_{2} \mathrm{O}_{5}(8.49 \%), \mathrm{SiO}_{2}(6.32 \%)$ and minor components are $\mathrm{Al}_{2} \mathrm{O}_{3}>\mathrm{Na} 2 \mathrm{O}>\mathrm{MgO}>\mathrm{Cl}$. The important constituents of CEM are alkaline earth metal oxides and hydroxides (for example, calcium oxide and calcium hydroxide $[\mathrm{CH}]$ ), calcium phosphate, and calcium silicate. CEM differs chemically from MTAs and PCs, phosphorous is the major component of CEM, whereas this element is close to the detection limit in MTAs and PCs. In contrast with MTA, CEM shows surface composition similar to surrounding dentin. Since $\mathrm{HA}$ is the main component of dentin, similarity between CEM cement and dentin might help the cementogenesis over it.

\subsection{Portland Cement [5]}

It is a calcium silicate product obtained by calcination of limestones. Though considered as an alternative to MTA it presents many limitations like it is radiolucent, non-sterile etc. But after overcoming these limitations it can have applications same as MTA.

\subsection{Ortho MTA [9]}

Recently, Ortho MTA (BioMTA, Seoul, Republic of Korea) was introduced for apex closure of an immature root, orthograde root canal filling, perforation repair, and retrograde filling. The manufacturer claims that Ortho MTA

\section{Volume 6 Issue 12, December 2017}




\section{International Journal of Science and Research (IJSR) \\ ISSN (Online): 2319-7064}

Index Copernicus Value (2015): 78.96 | Impact Factor (2015): 6.391

has similar components as ProRoot MTA but less heavy metal contents than ProRoot MTA.

\subsection{Mesocalicuim Silicate [10]}

Calcium Silicate based materials can promote hard tissue regeneration and have the ability to stimulate odontogenic and osteogenic differentiation in various types of cells, such as bone marrow stromal cells, adipose-derived stem cells, human dental pulp cells, and periodontal ligament cells However, this material has exhibited 2 weaknesses that the size of the CS-based material particles is usually at the micrometer level, making it difficult to inject, and traditional CS-based materials have a minimal nanopore structure, which greatly limits the potential for drug delivery. Hence, Mesoporous materials may be used as novel drug delivery carriers with release kinetics that can be controlled by adjusting hollow internal microstructures. By definition, a mesoporous material is a structure with pores that have diameters between 2 and $50 \mathrm{~nm}$; it is intermediate in size between microporous $(<2 \mathrm{~nm})$ and macroporous $(>50 \mathrm{~nm})$ materials. It has been suggested that structurally well-ordered mesoporous materials could potentially act as carriers for loading biomolecules and harmonizing their release. Targeted drug delivery is an important tumor therapy method, and the bioactivity of materials is of great importance for promoting tissue regeneration.

\subsection{Endocem [11]}

Recently, MTA-derived pozzolan cement was introduced which is fast setting without the addition of any acceleration because of the small sized pozzolan cement additives. It has properties similar to that of MTA but is faster setting which is helpful while endodntic surgeries.

\subsection{Others [12]}

Many other formulations are still under research like MTA Bio, Flouride-doped MTA, MTA sealer, Pro-root Endo sealer etc.

\section{Conclusion}

Calcium Silicate has proved to be a consistent material and researches going on to enhance its properties are proceeding in a right direction. Starting from MTA, calcium silicate has been continuously contributing to Endodontics.

\section{References}

[1] Parirokh M, Torabinejad M "Mineral Trioxide Aggregate: A Comprehensive Literature Review-Part I: Chemical, Physical, and Antibacterial Properties" Journal of Endodontics 2010 (36) pp. 16-27.

[2] Torabinejad M, Parirokh M, "Mineral Trioxide Aggregate: A Comprehensive Literature Review-Part II: Leakage and Biocompatibility Investigations." Journal of Endodontics 2010 (36) pp. 190-202.
[3] Tawil et al, "MTA: A Clinical Review." Compend Contin Educ Dent. 2015 (4): 247-264.

[4] Khan et al, "Mineral Trioxide Aggregate Use in Pediatric Dentistry: A Literature Review." Journal of Oral Hygiene and Health 2016, DOI: 10.4172/23320702.1000209 [Accessed Dec2016]

[5] Macwan et al, "Mineral trioxide aggregate (MTA) in dentistry: A review of literature" Journal of Oral Research and Review 2014 (6) pp71-74.

[6] Madfa et al, "Endodontic Repair Filling Materials: A Review Article." British Journal of Medicine \& Medical Research, 2014(4): 3059-3079.

[7] Sun Y et al, "Effect of iRoot Fast Set root repair material on the proliferation, migration and differentiation of human dental pulp stem cells in vitro." https://doi.org/10.1371/journal.pone.0186848 October 23, 2017 [Accessed Oct 2017]

[8] Utneja $\mathrm{S}$ et al, "Perspectives of bio-ceramic technology in endodontics: calcium enriched mixture cement review of its composition, properties and applications." http://dx.doi.org/10.5395/rde.2015.40.1.1 [Accessed Aug 2014].

[9] Chang et al, "Heavy Metal Analysis of Ortho MTA and ProRoot MTA" Journal of Endodontics 2010 (37) pp. 67-78

[10]Huang et al, "Mesoporous Calcium Silicate Nanoparticles with Drug Delivery and Odontogenesis Properties." Journal of Endodontics 2017 (43) pp 467 72.

[11] Choi Y. et al. "Biological effects and washout resistance of a newly developed fast setting pozzolan cement." Journal of Endodontics 2013 (39) pp 69-76.

[12] Huffman B P et al, "Dislocation resistance of ProRoot Endo Sealer, a calcium silicate-based root canal sealer, from radicular dentine." International Endodontic Journal 2009 (42) pp. 34-46.

Volume 6 Issue 12, December 2017 